\title{
Kassenpflicht für die Früherkennung des Kolonkarzinoms
}

\author{
Seit Juli 2013 wird die Kolonkarzinom-Früherkennung von der obligatorischen Kran- \\ kenpflegeversicherung für alle 50- bis 69-Jährigen bezahlt. Das Eidgenössische \\ Departement des Innern hat mit diesem Entscheid dem von der Krebsliga Schweiz \\ initiierten Leistungsantrag in Teilen zugestimmt. Die Forderung nach einem \\ systematischen Screening bleibt bestehen.
}

\section{Cornelia Schwager}

Fachmitarbeiterin

Prävention und Früherkennung, Krebsliga Schweiz

\section{Das Kolonkarzinom ist häufig}

Das Kolonkarzinom ist in der Schweiz mit rund 4100 Neuerkrankungen pro Jahr eine der häufigsten Krebserkrankungen. Die Diagnose wird häufig erst in fortgeschrittenem Stadium gestellt. In einem frühen Stadium erkannt, ist das Kolonkarzinom meistens heilbar.

\section{Zur Unterstützung bei der Beratung von Patienten hat die Krebsliga praktisches Informationsmaterial erarbeitet.}

\section{Literatur \\ 1 Krankenpflege-Leistungsver- ordnung (KLV, SR 832.112.31) Art. 12d lit. b Massnahmen zur frühzeitigen Erkennung von Krankheiten bei bestimmten Risikogruppen. \\ 2 Antrag auf Kostenübernahme des Kolon-Karzinoms durch die obligatorische Kranken- pflegeversicherung betreffend der Leistung Kolon Karzinom Programm, eingereicht von der Krebsliga Schweiz mit der Trägerschaft KKP (2011, 2012, 2013) \\ 3 Krankenpflege-Leistungsver- ordnung (KLV, SR 832.112.31) Art. 12e Bst.d. Massnahmen zur frühzeitigen Erkennung in der allgemeinen Bevölkerung.}

\section{Korrespondenz: \\ Cornelia Schwager \\ Krebsliga Schweiz \\ Effingerstrasse 40 \\ CH-3001 Bern \\ Tel. 0313899358}

cornelia.schwager[at]

krebsliga.ch

\section{Fehlende gesetzliche Grundlage als Antrieb}

In der obligatorischen Krankenpflegeversicherung (OKP) war die Kolonkarzinom-Früherkennung bis vor kurzem auf eng definierte Risikogruppen beschränkt [1]. Die Krebsliga Schweiz und weitere Organisationen haben im Jahr 2011 beim Eidgenössischen Departement des Innern (EDI) einen «Antrag auf Kostenübernahme durch die OKP für die Leistungen eines Kolon Karzinom Screening Programms (KKP)» eingereicht [2]. Das KKP soll zu einer vermehrten Erkennung des Kolonkarzinoms in einem frühen Stadium und zu einer Reduktion der Sterblichkeit beitragen. Gleichzeitig sollen die Qualitätssicherung und der Zugang zur Chancengerechtigkeit gefördert werden. Mitgetragen wird der Antrag vom Kollegium für Hausarztmedizin, den Schweizerischen Gesellschaften für Gastroenterologie und Viszeralchirurgie, der Magendarmliga Schweiz, pharmaSuisse und swiss cancer screening.

\section{Schritt in die richtige Richtung, Forderung für ein systematisches Programm bleibt} Seit dem 1. Juli 2013 ist die Kostenübernahme der Kolonkarzinom-Früherkennung für 50- bis 69-Jährige im Leistungskatalog der OKP geregelt. Die Versicherung übernimmt alle zwei Jahre die Kosten für die Untersuchung auf okkultes Blut im Stuhl (gemäss
Analysenliste) und die Koloskopie im Falle eines positiven Befunds, oder alle zehn Jahre eine Koloskopie [3]. Das EDI bewilligt diese Leistung allerdings ohne ein damit einhergehendes Programm vorauszusetzen und ohne Franchisenbefreiung (im Unterschied zur systematischen Brustkrebs-Früherkennung). Für den Fall, dass ein Kanton ein organisiertes Programm einführen will, wird das EDI jedoch prüfen, ob die Untersuchung mit Franchisenbefreiung durchgeführt werden kann. Kantonale Pilotprogramme sind von grosser Wichtigkeit, können damit doch Fragen zum systematischen Kolonkarzinom-Screening für die ganze Schweiz geklärt werden. Aus diesem Grund ist es unerlässlich, dass diese unterstützt und gefördert werden.

Die Krebsliga Schweiz und die Trägerschaft KKP erachten den Entscheid des EDI zugunsten einer besseren Kolonkarzinom-Früherkennung als Schritt in die richtige Richtung. Die Forderung nach einem systematischen Programm bleibt jedoch bestehen.

\section{Fachpersonen und Bevölkerung informieren}

Es dürfte noch einige Jahre dauern, bis ein systematisches Kolonkarzinom-Screening in der ganzen Schweiz eingeführt wird. Ärztinnen und Ärzte nehmen bis dahin eine umso wichtigere Rolle als Vertrauensperson ein, indem sie ihre Patientinnen und Patienten über die Möglichkeit der Früherkennung aufklären und sie bei der Wahl einer Untersuchungsmethode beraten und unterstützen. Frauen und Männer von 50 bis 69 Jahren sollen gut informiert ihre Entscheidung zur Früherkennung fällen und dabei von einer möglichst hohen Qualität der Untersuchungen profitieren können.

Zur Unterstützung der Fachpersonen bei der Beratung von Patientinnen und Patienten und als Entscheidungshilfe für Frauen und Männer hat die Krebsliga praktisches Informationsmaterial erarbeitet. Dieses steht den Fachpersonen und der Bevölkerung ab März, dem internationalen DarmkrebsMonat, unter www.krebsliga.ch/darmkrebs zur Verfügung. 